\title{
Biomechanics of insect cuticle: an interdisciplinary experimental challenge
}

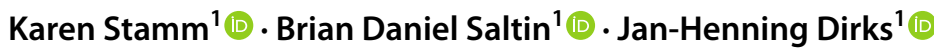

Received: 13 November 2020 / Accepted: 15 March 2021 / Published online: 11 April 2021

(c) The Author(s) 2021

\begin{abstract}
The cuticle exoskeleton plays a key role in facilitating the evolutionary success of insects. Since the mid of the last century, many different biomechanical properties of exoskeletons have been investigated, always utilizing the most sophisticated scientific methods available at the time. So far, information on the biomechanical properties of cuticle seems to be as diverse as the methods used to measure them. As a consequence, insect cuticle is often considered to exhibit the most complex and diverse biomechanical properties of any biological material. However, it remains unclear which role the respective measurement methods and sample treatments used in previous studies play in supporting this claim. This review provides a broad overview of examination techniques used to study biomechanical properties of insect exoskeletons and discusses their respective advantages and disadvantages in describing the properties of a complex material such as cuticle. Our meta-analysis of the present data confirms significant effects of the respective measurement methods, sample treatments and body parts on the obtained mechanical properties. Based on our findings, we highlight research gaps and point out important factors which should be taken into account in future studies on insect cuticle.
\end{abstract}

Keywords Material composition $\cdot$ Microstructure $\cdot$ Mechanical properties $\cdot$ Insect exoskeleton

\section{A complex material requires complex analysis}

After several decades with relatively slow progress in understanding the biomechanics of insect cuticle [1-6], significant advancements have been made in this field within the past years [7]. This growing interest in cuticle is most likely closely linked to two major factors: first, an increased appreciation and awareness for inter- and cross-disciplinary studies within research in organismal biology. These more holistic scientific approaches combine the fields of (cuticle) biomechanics with genetics, proteomics, material sciences, engineering and computing science to investigate fundamental properties of a complex biological material. Second, a growing access to affordable and yet powerful experimental and analytical techniques allows 'classic' organismal biologists, in particular from zoology and entomology, to use tools initially designed for mechanical testing to characterize

Jan-Henning Dirks

Jan-Henning.Dirks@hs-bremen.de

1 Biomimetics-Innovation-Centre, Hochschule Bremen-City University of Applied Sciences, 28199 Bremen, Germany biological materials. Easier access to improved high-resolution microscopy (e.g. X-ray microtomography, atomic force microscopy, confocal laser scanning microscopy) allows a more detailed insight into structure and geometry of complex biological samples [8].

Consequently, scientists from all kinds of disciplines publish their findings on different cuticle properties from various body parts across a variety of insect species, tested with several mechanical setups. However, there are no widely accepted 'standards' for biomechanical tests on insect cuticle (as there are for many other medical or biological materials such as bone, skin, wood, etc. [9-18]) and existing standards for nomenclature unfortunately differ between disciplines. Therefore, with a raising number of studies on insect cuticle, it becomes increasingly difficult to compare results, find similarities and draw general conclusions even in this relatively narrow scientific field.

A prominent feature of insect cuticle, stated in almost any paper written on this topic, is the huge range and variability of biomechanical properties of this complex biological composite material [1, 19-23]. Each new study seems to add a data point increasing this range and raising new questions. How can cuticle properties differ so strongly? 
Which role do different measurement techniques play in characterizing insect cuticle? Is there a 'gold standard' to study the biomechanical properties of cuticle? Also, cuticle is often considered as a good example for bio-inspirationally designed materials [24, 25]. However, what do we want to learn from cuticle if we still cannot draw a clear picture of its fundamental properties?

In this review, we first highlight and discuss several experimental challenges when analysing insect cuticle. We also summarize a representative selection of data (Tabl.1, [20-22, 26-58]) published on the biomechanical properties of insect exoskeleton. We have structured the data with respect to the experimental methods used to obtain the data (e.g. cantilever bending, nanoindentation, tensile testing) and statistically analysed if and how the choice of methods, insect species and body parts affects the results of the selected studies.

Obviously, this review cannot be comprehensive with respect to covering all values ever published on insect cuticle. Instead, we want to raise awareness of possible methodological problems when studying the biomechanics of insect cuticle, illustrate gaps in our knowledge and thus hopefully help to increase future compatibility and comparability between scientific studies and biomimetic projects working on this fascinating material.

\section{Structure of 'the' insect exoskeleton and cuticle}

Arthropod cuticle is one of the world's most common biological composite materials and one of the most evolutionary successful forms of 'outer body protection' [59]. Based on its outstanding evolutionary success and abundance in our ecosystem, the structure and composition of insect cuticle have been the biological inspiration for several biomimetic studies: For example, composite structures inspired by cuticle can increase strength in look-alike designed composite laminate with pore-like holes [24]. The manufacturing of paper-like waterproof and very high wet strength material has been inspired by the tanning of the cuticle's protein matrix [25]. Several aspects of the insect exoskeleton and its biomechanical properties have been studied to improve micro-air vehicles and other robotic systems [60-63]. In particular, walking robots have been inspired by insect exoskeletons and cuticle. For example, structural data on cockroach legs were used to manufacture insect-like legs from E-glass and S-glass, which were capable of optimal grip in various terrain $[64,65]$. One can certainly argue that entomology is an almost endless inspiration for biomimetics [66].

However, one of the first insights when studying arthropod exoskeletons and cuticle and using them as a template for bio-inspired solutions should be the fact that there is no such single thing as 'the' typical exoskeleton or cuticle. Like most biological materials, various types of cuticle have different origins, structure, function and chemical composition. In this study, within the group of Arthropod cuticles, we focus on the exoskeletal cuticle of adult insects, which already includes a huge variety of body parts with multiple functions and properties. Just looking at exoskeletal parts related to the insect wing illustrates this variety of biomechanical properties. Wing membranes for example need to be thin, lightweight and flexible, yet in combination with the veins they also add to the structural stability of a complex insect wing [67]. The wings of Odonata have a material Young's modulus of 2.74 GPa [55]. Beetle elytra, however, are used to protect the hind wings, but also need to be lightweight and flexible [42]. In beetle elytra, a reduced Young's modulus of $6.17 \mathrm{GPa}$ was measured [56]. Compared to the elytra, the Odonata wings Young's modulus is surprisingly low and may be more fitting for a highly flexible structure. Taking an even closer look at the wings, the wing veins are stiffer than the membrane to stop crack propagation. Here the mixture of a stiffer and a softer material (vein, membrane) and its respective function form a complex strong relationship [49, 68].

Insect wings are obviously only one of many examples for this versatility and multifunctionality of cuticle. Compound eyes of insects require excellent optical properties and transparency, while also providing sufficient physical protection and durability [69]. Some insect mandibles used for cutting plant material have highly sophisticated surface structures and are even reinforced with zinc and manganese [70]. As we will discuss in detail later, these selected examples already illustrate that the structure of the body part and thus the corresponding cuticle most likely plays an important role as it influences equally the function and mechanical properties. As osteologists will not just refer to 'bone' and carpenters will not just use 'wood', the biomechanics community should be careful in generalizing 'cuticle'.

\subsection{Structure or material constant?}

To report geometry-independent material constants of a structure such as an exoskeleton, it is important to be aware of both the mechanical behaviour (such as stress-strain relation) and the geometric features of the tested sample (such as cross section and second moment of area). It is relatively straightforward to measure forces and deflection in an experimental setup. However, it is much more challenging to characterize the correct geometry factor of a complex exoskeletal structure. As a consequence, many studies on insect cuticle (in particular from the pre-microCT age) have primarily focused on measuring and reporting structural features of exoskeletal parts only [71]. It is important to note that these studies are not representative for cuticle as 
biological material. Instead, these reports characterize features of a respective body part at a given load from a particular direction in high detail. The measured properties describe the structural behaviour and not the material properties of which the investigated structure is build. In particular for insect wings, where the geometric parameters of the complex vein and membrane structures are difficult to measure, values for a 'bending stiffness' are commonly reported [28, $29,34,52]$. In these studies, wings or sections of wings were often deflected and 'bending stiffness' was calculated using Hooke's law. When examining data published on insect cuticle biomechanics, it needs to be stressed how important it is not to mix these values reported in $\mathrm{Nm}^{-1}$ with measurements of actual 'flexural stiffness', which are usually reported in $\mathrm{Nm}^{-2}$. The latter takes the whole structure into account, while 'bending stiffness' in $\mathrm{Nm}^{-1}$ describes the force required to move the structure for a certain length. However, in the right context, these measurements of structural characteristics of insect body parts do have their value for biologists or biomimetics researchers. A material value obtained through suitable mechanical measurements provides information about cuticle as a material only, disregarding its biological function. Thus, a Young's modulus reported without any further context can be 'surprisingly low' for a material. If used in a specific morphology, however, a low Young's modulus can result in completely different biological conclusions [72, 73]. Discussing a structural value (material and morphology) of an entire exoskeleton body part, however, provides comprehensive information about the biomechanical in situ behaviour of a complex and multifunctional structure. Therefore, reporting structural values can be of particular interest for wings, joints and other dynamic and highly complex skeletal elements.

As a consequence, reporting flexural stiffness for insect cuticle samples without sufficient context has rather limited scientific value. Considering the increasing availability of non-destructive methods to characterize a sample's geometry at high precision (such as X-ray microtomography), great care should be taken to only report structural parameters when this approach is clearly justified and no better experimental option is available.

Interestingly, our literature survey shows that there is almost no comprehensive data set for structural and material values of the same body part in the same insect species available. Our analysis showed that certain taxonomic groups are investigated way more frequently regarding either their material or their structural properties (Fig. 1).

\subsection{Young's modulus as a measure of cuticle properties?}

The Young's modulus or elastic modulus (E) provides information about the stress-strain relationship as a geometry-independent material constant. Knowing the Young's modulus of a biological material and linking it to other biomechanical properties may lead to a better understanding

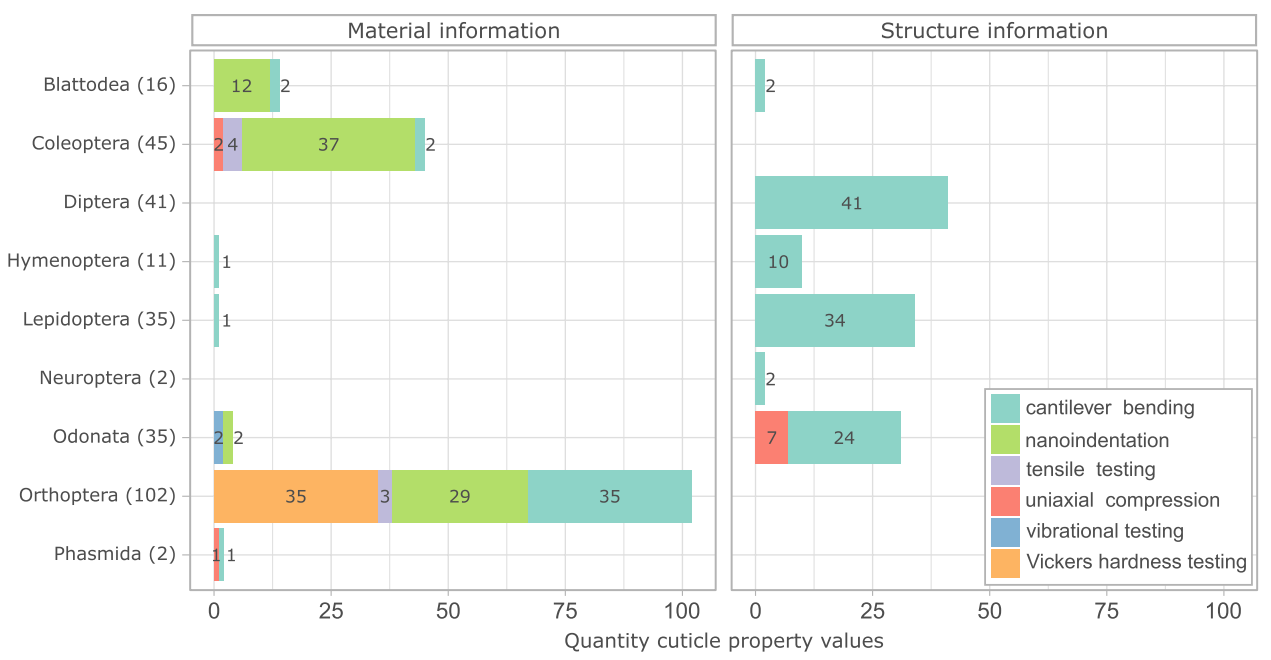

Fig. 1 Distribution of information on 'material' and 'structure' properties of insect cuticle in different taxonomic groups. This figure shows that within the data set of this study insect taxonomic groups seem to be mostly investigated regarding either their structural or their material properties. For only four taxonomic groups, both material and structural information is available (Blattodea, Hymenoptera, Lepidoptera, Odonata). Furthermore, the analysis of the present data shows that cantilever bending and nanoindentation often seems to be the methods of choice to investigate insect cuticle properties. Bars represent absolute number of data points in our data set of publications. Numbers behind the taxonomic groups indicate total number of data points, numbers in the bars show data points obtained with the respective method. The colours indicate the used method: cantilever bending (turquoise), nanoindentation (green), tensile testing (purple), uniaxial compression (red), vibrational testing (blue) and Vickers hardness testing (orange). The complete data set of this study is available as suppl. material 
of structure-function correlations [45]. Labonte et al. [74], for example, pointed out that in insect mandibles material properties of the elastic modulus and the indentation hardness are linked. The indentation hardness of cuticle can thus be seen as a 'hybrid property' to quantify material reversible and irreversible deformation.

One of the main challenges in reporting Young's moduli of a hierarchically organized, anisotropic composite material is that a specific modulus is only applicable in the linear elasticity region of a uniaxial deformation curve. These features are obviously difficult to find in complex stress-strain curves from biological samples, in particular insect cuticle [3]. Thus, reporting Young's moduli for insect cuticle should ideally include a precise description of the sample orientation, sample ultrastructure and the applied stresses (e.g. speed of deflection, direction of applied stress).

The reported range of elastic modulus in unspecified 'cuticle samples' covers several orders of magnitude [23]. Our analysis confirms that values of Young's moduli reported for sclerotized insect cuticle samples using a variety of methods are in a range from 1.2 MPa to $10.9 \mathrm{GPa}$ (Fig. 2). The smallest value was measured using nanoindentation on a fresh tarsal setae sample of a Coccinella septempuncta [47]. These cuticular structures are used for attachment during locomotion and require a complex combination of rather compliant material properties to closely make contact to any surface. The highest Young's modulus was also measured using nanoindentation; however on a dry sternal plate of locust, a structure providing physical protection of the insect
[46]. These findings highlight not only the possible measurement range of nanoindentation as well as the importance of sample condition and examined body part.

Besides 'classic' mechanical tests to obtain values for the Young's modulus, Eshghi et al. [75] recently developed a qualitative non-destructive, unique method to estimate the elastic modulus of cuticle by linking mechanical properties to the autofluorescence of cuticle material components (e.g. resilin dominated cuticle, less-sclerotized cuticle and highly sclerotized cuticle). This method can be valuable considering material gradients within a structure and can help to visualize and analyse these functions and material properties by detecting locations of different types of cuticle [72].

Regardless of the measurement technique, it is important to emphasize the often complex interactions between Young's modulus and other biomechanical parameters, such as strength, fracture toughness and hardness. Reporting the Young's modulus of cuticle alone only tells a very small part of the overall story and drawing biological or biomechanical conclusions from this 'cuticle material constant' should be well justified.

\section{Effect of measurement techniques}

Although in theory obtaining characteristic material properties should be independent from the respective method, the experimental reality will result in notable technique-dependent variation. To investigate and illustrate the possible effect
Fig. 2 Effect of measurement technique on Young's moduli (GPa) of insect cuticle. This data illustrates the high diversity of insect cuticle as well as the range of cuticle within a taxonomic group. Five different measurement techniques were used to obtain the elastic moduli: cantilever bending (turquoise), nanoindentation (green), tensile testing (purple), uniaxial compression (red) and vibrational testing (blue). A square indicates a sample size less than five, a dot indicates outliers. Sample treatment and body parts were summarized to their taxonomic group. As the Young's moduli range over several orders of magnitude the plot is separated into two subplots. Boxplots show median and quartiles following McGill et al. (1978) [102].

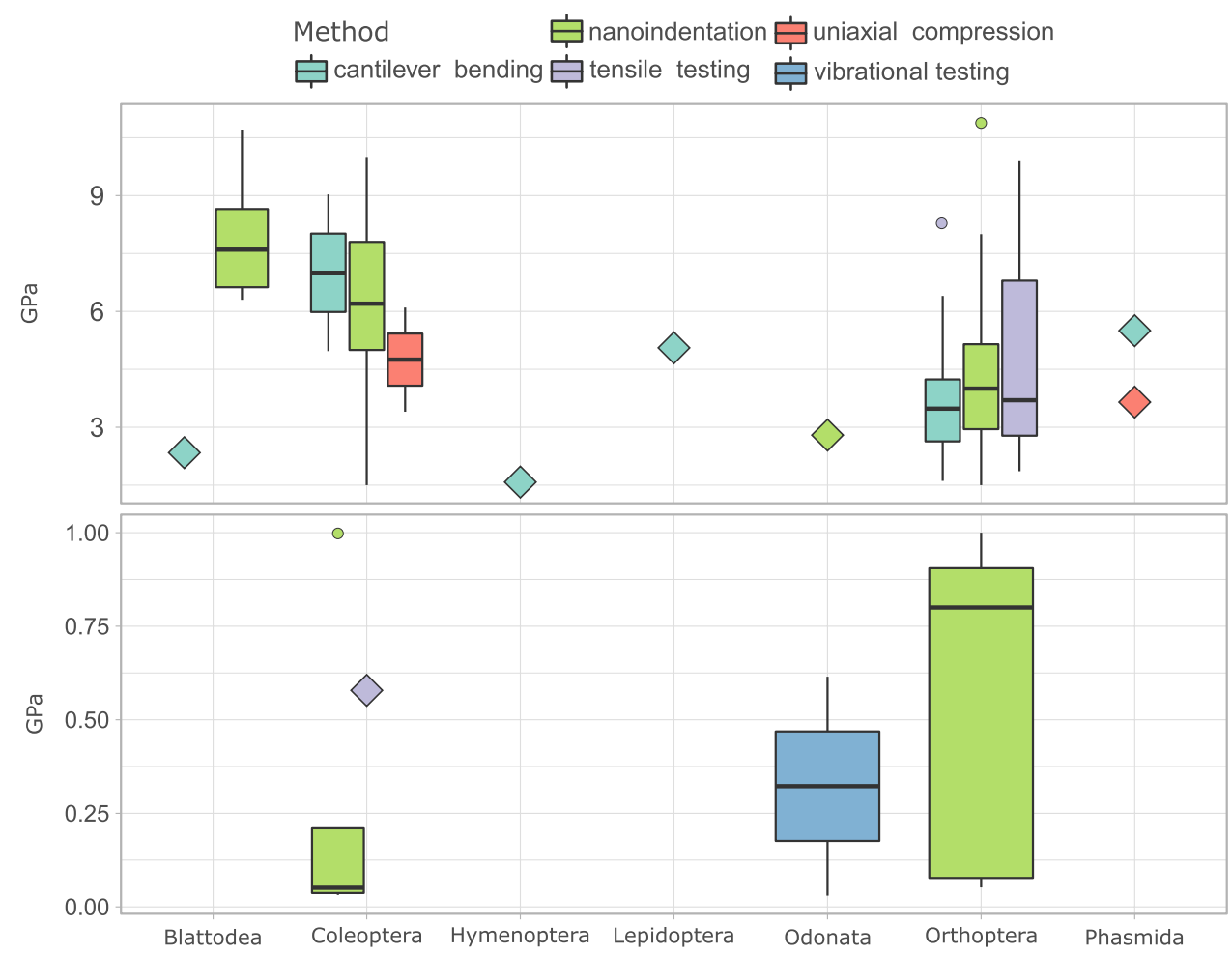


of measurement technique on Young's modulus, we statistically analysed our data with respect to the effect of the experimental methods (all analyses performed using R Studio [76]). If the geometry-independent Young's modulus would be the same across all types of cuticle, independent of any other factor, there should be no significant effect across our Young's modulus data set.

For our analysis, we split the obtained data into five groups of measurement techniques (cantilever bending, nanoindentation, tensile testing, uniaxial compression, vibration testing). Since Young's modulus deviates from normal distribution (Shapiro-Wilk test, $W=0.961, p<0.001$ ), we used a nonparametric Kruskal-Wallis test. Our results show a clear significant effect of the measurement technique on the reported values for Young's modulus of insect cuticle $\left.\left(\chi^{2}(4)=9.723, p<0.05\right)\right)$. In addition, this result also statistically supports the fact that there is no such thing as 'the' Young's modulus for insect cuticle.

More detailed data on measurement direction, sample treatment and growth condition could explain additional variations between the measured material properties in one particular body part. For flexural stiffness and bending stiffness of cuticle, only very little data are available and thus the results should be treated with caution. Given the sparsely available data, a further statistical analysis (for example including a multi-factor model) would be possible, however not meaningful.

\section{Effect of sample treatment}

Apart from material composition and microstructure, the age of the samples and growth conditions can strongly influence cuticle properties $[23,77]$. Several recent studies have clearly shown that, as with most biological materials, cuticle samples are very sensitive to test conditions, in particular the water content and chemical treatment (tanned, dry, fresh, rehydrated, etc.) [20, 46, 48, 74, 78, 79].

To investigate the possible effect of sample treatment on Young's modulus and flexural stiffness across all measurement techniques, we split our data into nine groups of sample treatment (chemical, dry, dry-chemical, fresh, fresh-chemical, frozen, hydrated, medium, mixed). 'Chemical' sample treatment indicated the use of any chemical on the sample which is not rehydrating the sample; 'mixed' describes the usage of multiple sample treatments for which only one value was reported. In 'fresh' samples, measurements were taken within 45 min after dissection; 'medium' samples were measured between 45 and 60 min. 'Dry' samples were measured more than $60 \mathrm{~min}$ after dissection or desiccated on purpose. Our analysis showed that both Young's modulus and flexural stiffness are significantly affected by the sample treatment $\left(\chi^{2}(8)=44.964, p<0.001 ; \chi^{2}(3)=69.679\right.$, $p<0.001$; Kruskal-Wallis test). This result again is not particularly surprising, as for several examples, such as locust hind leg tibiae, it has been shown that desiccation significantly increases the Young's modulus and bending strength while decreasing the fracture toughness [48]. Thus, in each sample the possible function, shape, water content and additional treatment should be considered during any investigation. This is of particular importance for measurement techniques such as nanoindentation, which often requires an elaborate sample pre-treatment (embedding, polishing, etc.).

\section{Differences between body parts and taxonomic groups}

As stated above, mechanical properties and sample geometry need to be analysed together as they show complex interaction [5, 6, 9, 23, 45, 80, 81]. However, even when only material values are reported, variations within one single body part across different species can be observed, especially if the cuticle is reinforced with minerals ( $\mathrm{Zn}, \mathrm{Mn}$ or $\mathrm{Fe}$ ) to increase hardness and resistance to wear [82].

As already summarized in Li et al. [22], the elastic moduli for insect hind tibiae ranged from 1.3 to $9 \mathrm{GPa}$. Regarding only data from one species (Schistocerca gregaria) and the same body part (hind leg tibia), the data still varied between 3 and $9 \mathrm{GPa}[1,20,22,31,45,77]$. The lower elastic modulus was measured by compression and by using a fresh tibia [20]. On the other hand, the highest value (9 GPa) was also measured by compression but using a dry tibia [1]. Other elastic moduli of locust hindleg tibia within this range was measured in dry or fresh state by using nanoindentation, compression and bending test $[1,20,22]$.

Indeed, our statistical analysis shows a significant effect of the tested body part on the obtained values for Young's modulus $\left(\chi^{2}(13)=63.027, p<0.001\right)$ and flexural stiffness $\left(\chi^{2}(10)=75.483, p<0.001\right)($ Fig. 3).

Properties of unidirectional composite materials at one point within a specimen are not necessarily the same in every direction $[23,83]$. Unidirectional layers can influence material properties in more than one stress direction, and thus, the direction of measurement very often significantly affects the measured mechanical properties [84]. Hence, within one body part the direction of measurement can cause a huge variety in the measured results. Materials with an inner, more compliant layer and an outer layersuch as cuticle_-show a different deformation behaviour before material failure due to an increase of elastic energy storage depending on the measurement direction [23, 85]. The outer layer will be compressed while the inner layer is under tension when a beam load is applied from the direction of the outer layer. Thus, for a given body part, cantilever bending results might show other aspects of material 
Fig. 3 Flexural stiffness $\left(\mathrm{Nm}^{-2}\right)$ of insect cuticle in different taxonomic groups measured using cantilever bending. Sample treatment and body parts were summarized to their taxonomic group. Dots indicate outliers, boxplots show median and quartiles following McGill et al. (1978) [102]

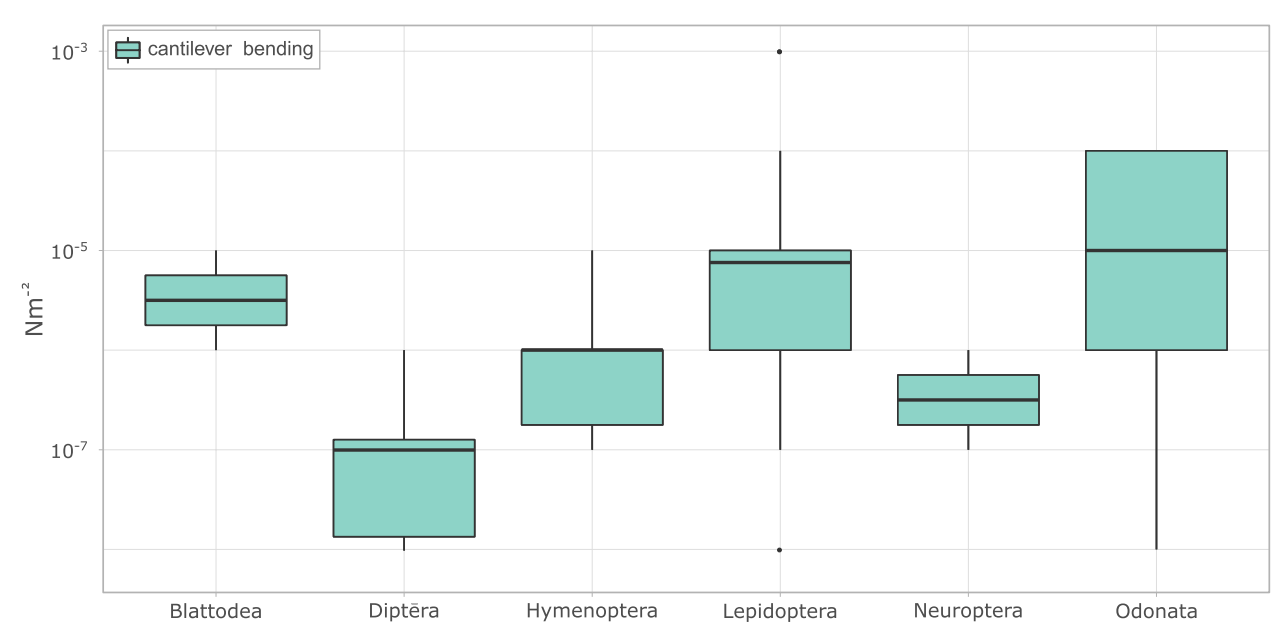

behaviour than for example nanoindentation, where the technique might be penetrating only one layer at a time. In nanoindentation, the samples general shape is not influencing the measurement and it is possible to measure in such detail that the body part's fibre alignment may have already a notable impact on the results.

Even though a variety of different insect taxonomic groups were already investigated, it is unfortunately very rare that a single species is investigated using different methods. Only four out of eleven taxonomic groups were investigated regarding both structural and material properties of cuticle (Blattodea, Hymenoptera, Lepidoptera, Odonata, see Fig. 1). Here, cantilever bending is frequently used to determine material and structural cuticle properties. Our statistical analysis shows a significant effect of taxonomic group on Young's modulus $\left(\chi^{2}(6)=26.369, p<0.001\right)$ and flexural stiffness $\left(\chi^{2}(6)=91.868, p<0.001\right)$. Although given the relatively small sample size, all statistical results need to be treated with great caution. Nonetheless, together these findings highlight once again the importance of specimen condition, test method, sample orientation and body part.

In addition, several studies indicate that cuticle might not necessarily be a 'static' material. Like many other biological materials, cuticle could react to external stimuli on different time scales. Short-term viscoelastic responses of cuticle to mechanical loads have been reported in various studies [3, $86,87]$ and have not yet received systematic attention. Reynolds for example stated that cuticle may be reinforced by chitin microfibrils shortly after imposition of strain [3]. This might lead to the assumption that measured high moduli could be 'short-term' behaviour of the material or structure, and cuticle may behave differently under long-time imposed stress [88]. This goes along with results of Dirks et al. [89], where fatigue tests have shown different failure reactions of locust wings and legs [89].
In several insect species, it has also been shown that in the first days and weeks post-molt the insects deposit additional layers cuticle $[90,91]$. This cuticle deposition is assumed to reinforce the exoskeleton [88]. As stated by Parle et al. [88], it would therefore be important to determine the cuticle deposition rate and possible related change in material properties. Also, if and how cuticle can react to mechanical stress-especially during its deposition time- remains unknown. If this holds true, it would introduce and require 'environmental mechanical stress' as an entirely new factor when characterising insect cuticle.

\section{Conclusion}

Alike studies on all complex biological materials, biomechanical measurements on insect cuticle are a challenging interdisciplinary task. Our meta-analysis quite clearly shows that there is a lack of comprehensive studies on insect cuticle, in which microstructure, sclerotization level and material properties are simultaneously investigated [47, 53, 92, 93]. The few more 'comprehensive' studies were often conducted on different insect's body parts with various sizes, ages, sexes, life histories, nutritional needs and environmental conditions [31, 37, 94, 95].

By highlighting all these aspects in this review, we want topoint out remaining open, fundamental questions regarding insect cuticle. These questions include the complex interaction of chitin fibres within the protein matrix, their effect on mechanical properties and the insect's ability to control these mechanisms (on a micrometre level and to build gradients within the material) [74, 96-98]. Another area of future research should be the mechanistic basis for the influence of hydration on toughness, Young's modulus and hardness [74]. In addition, several 'basic mechanical properties' of insect cuticle such as plasticity, yield, wear and fatigue properties remain mostly unknown [99-101]. Along with insect's 
ontogenesis, developmental stage (including the age), effects of environmental conditions (such as higher mechanical stress) and degree of sclerotization, these factors may play an important role as well.

Studies focusing on these questions may lead to advanced knowledge about range of cuticle mechanical properties and thus to a better understanding of function-properties-correlations and developmental and evolutionary biology of insects. We believe that when approaching these questions and characterising insect cuticle exoskeletons in particular, the following points should be considered in future studies:

- As with almost all biological materials, sample treatment can strongly affect all kinds of mechanical properties of cuticle. Several methods also require a specific sample treatment, which again can inevitably affect the properties of cuticle. The conditions of the cuticle sample (degree of hydration, chemical treatment, etc.) need to be monitored, documented and clearly stated for all measurements.

- As with all complex composite materials, the choice of measurement method can strongly affect the measured properties of cuticle. The chosen method to characterise the cuticle samples needs to be justified and its advantages and disadvantages need to be clearly discussed. Unfortunately, to our knowledge, there is no sufficient suitable comparable data set available within the scientific literature to evaluate the influence of method and sample treatment on cuticle in more detail.

- Particularly when analysing cuticle exoskeletons, it is important to clearly differentiate between material and structural properties and use neither of these values without biological context. Material and structural properties are depended on the method (and sample analysis) and provide different information about the sample. Meaningful property-function conclusions in cuticle exoskeletons can only be drawn when this issue is clearly addressed.

Insects are without doubt a fascinating and diverse taxon with the largest number of species. They go through multiple conecutive life stages as eggs, larvae, nymphs and adults, which often are facing very different environments. These different requirements are likely to involve various different cuticle 'configurations' and corresponding material properties. In contrast, researchers working in insect biomechanics are a very small scientific community. It is a certainly impossible task for this scientific community to try to comprehensively characterize a meaningful number of insect species to get a clear picture. Our literature survey indeed illustrates that there are very few comparative data available on cuticle samples from either multiple measurement sites of a single species, similar body parts of different species or even comparable experimental techniques. It might be tempting (and academically rewarding) to study cuticle in a particular interesting, rare species of insects. However, to contribute towards the general understanding of insect cuticle biomechanics, our community should try to focus our studies more often on several key species instead. Painting a broader picture and pointing out specific features within this fascinating and diverse group of animals is only scientifically meaningful if we first understand the general principles of insect cuticle.

Supplementary Information The online version contains supplementary material available at https://doi.org/10.1007/s00339-021-04439-3.

Authors' contribution K.S., B.S., J.-H.D. contributed to conceptualization; K.S. contributed to formal analysis; K.S. contributed to investigation; K.S. contributed to data curation; K.S. contributed to writingoriginal draft; K.S., B.S., J.-H.D. contributed to writing-review and editing; K.S. contributed to visualization; B.S., J.-H.D. contributed to supervision; J.-H.D. contributed to project administration.

Funding Open Access funding enabled and organized by Projekt DEAL. This work was supported by DFG Project 395713517 (JHD). B.S. is funded by the HSB Research Fellowship.

Data availability All data generated or analysed during this study are included in this published article and its supplementary information files.

\section{Declarations}

Conflicts of interest The authors have no conflicts of interest to declare that are relevant to the content of this article.

Open Access This article is licensed under a Creative Commons Attribution 4.0 International License, which permits use, sharing, adaptation, distribution and reproduction in any medium or format, as long as you give appropriate credit to the original author(s) and the source, provide a link to the Creative Commons licence, and indicate if changes were made. The images or other third party material in this article are included in the article's Creative Commons licence, unless indicated otherwise in a credit line to the material. If material is not included in the article's Creative Commons licence and your intended use is not permitted by statutory regulation or exceeds the permitted use, you will need to obtain permission directly from the copyright holder. To view a copy of this licence, visit http://creativecommons.org/licenses/by/4.0/.

\section{References}

1. M. Jensen, T. Weis-Fogh, J.W.S. Pringle, Philos. Trans. R. Soc. Lond. B. Biol. Sci. 245, 137 (1962)

2. S. O. Andersen, T. Weis-Fogh, in Adv. Insect Physiol., edited by J. W. L. Beament, J. E. Treherne, and V. B. Wigglesworth (Academic Press, 1964), pp. 1-65

3. S.E. Reynolds, J. Exp. Biol. 62, 69 (1975)

4. S.N. Gorb, Naturwissenschaften 86, 552 (1999)

5. M. F. Ashby, Materials and design: the art and science of material selection in product design, Butterworth-Heinemann (2013)

6. P. Weaver, M. Ashby, J. Eng. Des. 7, 129 (1996) 
7. B. Moussian, in Arthropod Biol. Evol., edited by A. Minelli, G. Boxshall, and G. Fusco (Springer Berlin Heidelberg, Berlin, Heidelberg, 2013), pp. 171-196

8. P.D. Lösel, T. van de Kamp, A. Jayme, A. Ershov, T. Faragó, O. Pichler, N. Tan Jerome, N. Aadepu, S. Bremer, S.A. Chilingaryan, M. Heethoff, A. Kopmann, J. Odar, S. Schmelzle, M. Zuber, J. Wittbrodt, T. Baumbach, V. Heuveline, Nat. Commun. 11, 5577 (2020)

9. U. G. K. Wegst, The mechanical performance of natural materials, $\mathrm{PhD}$ thesis, University of Cambridge, 1996

10. S. Amada, T. Munekata, Y. Nagase, Y. Ichikawa, A. Kirigai, Y. Zhifei, J. Compos. Mater. 30, 800 (1996)

11. S.C. Lakkad, J.M. Patel, Fibre. Sci. Technol. 14, 319 (1981)

12. J.S. Jennings, N.H. Macmillan, J. Mater. Sci. 21, 1517 (1986)

13. H. Oxlund, J. Manschot, A. Viidik, J. Biomech. 21, 213 (1988)

14. U. Bosch, B. Decker, W. Kasperczyk, A. Nerlich, H.-J. Oestern, H. Tscherne, J. Biomech. 25, 821 (1992)

15. G.J. Rogers, B.K. Milthorpe, A. Muratore, K. Schindhelm, Biomaterials 11, 89 (1990)

16. R.B. Ashman, J.Y. Rho, J. Biomech. 21, 177 (1988)

17. R. Dickenson, W. Hutton, J. Stott, Bone Joint Surg. Br. 63-B, 233 (1981)

18. D.D. Moyle, M.W. Walker, J. Biomech. 19, 613 (1986)

19. S. Büsse, S.N. Gorb, R. Soc, Open Sci. 5, 172117 (2018)

20. J.-H. Dirks, D. Taylor, J. Exp. Biol. 215, 1502 (2012)

21. F.-O. Lehmann, S. Gorb, N. Nasir, P. Schutzner, J. Exp. Biol. 214, 2949 (2011)

22. C. Li, S.N. Gorb, H. Rajabi, Acta Biomater. 103, 189 (2020)

23. J.F.V. Vincent, U.G.K. Wegst, Arthropod Struct. Dev. 33, 187 (2004)

24. B. Chen, X. Peng, W. Wang, J. Zhang, R. Zhang, Micron 33, 571 (2002)

25. M. Miessner, M.G. Peter, J.F.V. Vincent, Biomacromol 2, 369 (2001)

26. J. Zhang, G. Tan, M. Zhang, D. Jiao, Y. Zhu, S. Wang, Z. Liu, D. Liu, Z. Zhang, J. Mech. Behav. Biomed. Mater. 91, 278 (2019)

27. N. Barbakadze, J. Exp. Biol. 209, 722 (2006)

28. R. Ganguli, S. Gorb, F.-O. Lehmann, S. Mukherjee, S. Mukherjee, Exp. Mech. 50, 1183 (2010)

29. Y. Ma, H. Ren, J. Ning, P. Zhang, J. Bionic Eng. 14, 111 (2017)

30. J.K. Oh, S.T. Behmer, R. Marquess, C. Yegin, E.A. Scholar, M. Akbulut, Acta Biomater. 62, 284 (2017)

31. M. Schmitt, T.H. Büscher, S.N. Gorb, H. Rajabi, J. Exp. Biol. 221, jeb173047 (2018)

32. Y.H. Chen, M. Skote, Y. Zhao, W.M. Huang, Mater. Lett. 97, 166 (2013)

33. S.J. Steppan, J Res Lepid 35, 61 (2000)

34. H.-N. Wehmann, L. Heepe, S.N. Gorb, T. Engels, F.-O. Lehmann, Biol. Open 8, 038299 (2019)

35. S.A. Combes, T.L. Daniel, J. Exp. Biol. 206, 2989 (2003)

36. S.A. Combes, T.L. Daniel, J. Exp. Biol. 206, 2979 (2003)

37. Y. Matsumura, A.E. Kovalev, S.N. Gorb, Sci. Adv. 3, 5469 (2017)

38. J. Sun, J. Tong, and Zhijun Zhang, in 2009 Int. Conf. Mechatron. Autom. (2009), pp. 4277-4282

39. B.W. Cribb, A. Stewart, H. Huang, R. Truss, B. Noller, R. Rasch, M.P. Zalucki, Naturwissenschaften 95, 17 (2007)

40. T.E. Mengesha, R.R. Vallance, R. Mittal, Bioinspir. Biomim. 6, 014001 (2011)

41. J.E. Hillerton, S.E. Reynolds, J.F.V. Vincent, J. Exp. Biol. 96, 45 (1982)

42. J. Lomakin, P.A. Huber, C. Eichler, Y. Arakane, K.J. Kramer, R.W. Beeman, M.R. Kanost, S.H. Gehrke, Biomacromol 12, 321 (2011)

43. H.-T. Lee, H.-J. Kim, C.-S. Kim, K. Gomi, M. Taya, S. Nomura, S.-H. Ahn, Acta Biomater. 57, 395 (2017)
44. Z. Dai, Z. Yang, J. Bionic Eng. 7, 6 (2010)

45. E. Parle, S. Herbaj, F. Sheils, H. Larmon, D. Taylor, Bioinspir. Biomim. 11, 016003 (2015)

46. D. Klocke, H. Schmitz, Acta Biomater. 7, 2935 (2011)

47. H. Peisker, J. Michels, S.N. Gorb, Nat. Commun. 4, 1661 (2013)

48. B. Aberle, R. Jemmali, J.-H. Dirks, Arthropod Struct. Dev. 46, 138 (2017)

49. J.-H. Dirks, D. Taylor, PLoS ONE 7, e43411 (2012)

50. C.M. Hayot, S. Enders, A. Zera, J.A. Turner, J. Mater. Res. 28, $2650(2013)$

51. L. Li, C. Guo, S. Xu, X. Li, C. Han, Microsc. Res. Tech. 80, 704 (2017)

52. N. Matushkina, S. Gorb, Zoology 110, 167 (2007)

53. M. Müller, M. Olek, M. Giersig, H. Schmitz, J. Exp. Biol. 211, $2576(2008)$

54. M. O’Neill, D. Taylor, J. Exp. Zool. Part Ecol. Integr. Physiol. 333, $20(2020)$

55. F. Song, K.W. Xiao, K. Bai, Y.L. Bai, Mater. Sci. Eng. A 457 , $254(2007)$

56. J. Sun, J. Tong, J. Bionic Eng. 4, 11 (2007)

57. C.W. Smith, R. Herbert, R.J. Wootton, K.E. Evans, J. Exp. Biol. 203, 2933 (2000)

58. S.S. Singh, M.A. Jansen, N.M. Franz, N. Chawla, Mater. Charact. 118, 206 (2016)

59. Z. W. White and F. J. Vernerey, Bioinspir Biomim 21 (2018)

60. K.Y. Ma, P. Chirarattananon, S.B. Fuller, R.J. Wood, Science 340, 603 (2013)

61. H. Liu, S. Ravi, D. Kolomenskiy, H. Tanaka, Philos. Trans. R. Soc. B Biol. Sci. 371, 20150390 (2016)

62. R. Altendorfer, N. Moore, H. Komsuoglu, M. Buehler, H.B. Brown, D. McMordie, U. Saranli, R. Full, D.E. Koditschek, Auton. Robots 11, 207 (2001)

63. H. Witte, H. Hoffmann, R. Hackert, C. Schilling, M.S. Fischer, H. Preuschoft, J. Anat. 204, 331 (2004)

64. S. F. Frazier, G. S. Larsen, D. Neff, L. Quimby, M. Carney, R. A. DiCaprio, and S. N. Zill, J. Comp. Physiol. [A] 185, 157 (1999)

65. C. Prahacs, A. Saudners, M.K. Smith, D. McMordie, M. Buehler, Proc (Can. Eng. Educ. Assoc, CEEA, 2011).

66. S. N. Gorb, in Insect Biotechnol., edited by A. Vilcinskas (Springer Netherlands, Dordrecht, 2011), pp. 241-264

67. P. Kreuz, W. Arnold, A.B. Kesel, Ann. Biomed. Eng. 29, 1054 (2001)

68. H. Rajabi, D. A., S. A., T. D., and D. J.-H., J. Biomech. 48, 89 (2015)

69. H. Peisker, S.N. Gorb, J. Exp. Biol. 213, 3457 (2010)

70. D.L.J. Quicke, P. Wyeth, J.D. Fawke, H.H. Basibuyuk, J.F.V. Vincent, Zool. J. Linn. Soc. 124, 387 (1998)

71. D.J.S. Newman, R.J. Wootton, J. Exp. Biol. 125, 361 (1986)

72. J. Michels, S.N. Gorb, J. Microsc. 245, 1 (2011)

73. B.D. Saltin, Y. Matsumura, A. Reid, J.F. Windmill, S.N. Gorb, J.C. Jackson, Insects 11, 520 (2020)

74. D. Labonte, A.-K. Lenz, M.L. Oyen, Acta Biomater. 57, 373 (2017)

75. Sh. Eshghi, M. Jafarpour, A. Darvizeh, S.N. Gorb, H. Rajabi, J. R. Soc. Interf. 15, 20180312 (2018)

76. T. RStudio, RStudio: Integrated Development for $R$ (PBC, Boston, MA, 2020)

77. E. Parle, D. Taylor, J. Mech. Behav. Biomed. Mater. 68, 247 (2017)

78. E. Arzt, S. Gorb, R. Spolenak, Proc. Natl. Acad. Sci. 100, 10603 (2003)

79. S. Enders, N. Barbakadse, S.N. Gorb, E. Arzt, J. Mater. Res. 19, 880 (2004)

80. P.M. Weaver, M.F. Ashby, Mater. Limits Shape Effic. 41, 61 (1997) 
81. U.G.K. Wegst, M.F. Ashby, Philos. Mag. 84, 2167 (2004)

82. A. Dieterich and O. Betz, Elementsensitive Synchrotron-Mikrotomographie zur Darstellung von Zinkeinlagerungen in den Mandibeln ausgewählter Insekten, Mitteilungen der Deutschen Gesellschaft für allgemeine und angewandte Entomologie. 17, 285-288 (2009)

83. J.F.V. Vincent, Tissue Cell 13, 831 (1981)

84. A.C. Orifici, I. Herszberg, R.S. Thomson, Compos. Struct. 86, 194 (2008)

85. R. F. Ker, Some structural and mechanical properties of locust and beetle cuticle, PhD thesis, University of Oxford, 1977

86. R. Blickhan, J. Biomech. 19, 375 (1986)

87. T.G. Bayley, G.P. Sutton, M. Burrows, J. Exp. Biol. 215, 1151 (2012)

88. E. Parle, J.-H. Dirks, D. Taylor, Arthropod Struct. Dev. 46, 49 (2017)

89. J.-H. Dirks, E. Parle, D. Taylor, J. Exp. Biol. 216, 1924 (2013)

90. E. Parle, J.-H. Dirks, D. Taylor, J.R. Soc, Interface 13, 20150984 (2016)

91. L. Hill, G.J. Goldsworthy, J. Insect Physiol. 14, 1085 (1968)

92. P.P. Goodwyn, A. Peressadko, H. Schwarz, V. Kastner, S. Gorb, J. Comp. Physiol. A 192, 1233 (2006)

93. I. Scholz, W. Baumgartner, W. Federle, J. Comp. Physiol. A 194, $373(2008)$
94. C. Wan, Z. Hao, X. Feng, Sci. Rep. 6, 35219 (2016)

95. L.-Y. Wang, H. Rajabi, N. Ghoroubi, C.-P. Lin, and S. N. Gorb, Front. Physiol. 9, 1410 (2018)

96. S. Amini, M. Tadayon, S. Idapalapati, A. Miserez, Nat. Mater. 14, 943 (2015)

97. F. Barthelat, H.D. Espinosa, Exp. Mech. 47, 311 (2007)

98. F. Barthelat, H. Tang, P.D. Zavattieri, C.-M. Li, H.D. Espinosa, J. Mech. Phys. Solids 55, 306 (2007)

99. H.S. Gupta, J. Seto, W. Wagermaier, P. Zaslansky, P. Boesecke, P. Fratzl, Proc. Natl. Acad. Sci. 103, 17741 (2006)

100. H.S. Gupta, S. Schratter, W. Tesch, P. Roschger, A. Berzlanovich, T. Schoeberl, K. Klaushofer, P. Fratzl, J. Struct. Biol. 149, 138 (2005)

101. J. Keckes, I. Burgert, K. Frühmann, M. Müller, K. Kölln, M. Hamilton, M. Burghammer, S.V. Roth, S. Stanzl-Tschegg, P. Fratzl, Nat. Mater. 2, 810 (2003)

102. R. McGill, J.W. Tukey, W.A. Larsen, Am. Stat. 32, 12 (1978)

Publisher's Note Springer Nature remains neutral with regard to jurisdictional claims in published maps and institutional affiliations. 